\title{
SOBRE LA BÚSQUEDA DE LA NATURALEZA JURÍDICA. UN COMENTARIO A PROPÓSITO DE LA COMPENSACIÓN ECONÓMICA (SENTENCIA DE LA CORTE SUPREMA)
}

\author{
Comentario de David Quintero Fuentes
}

Santiago, veintisiete de abril de dos mil nueve.

VISTOS:

En estos autos, RIT N ${ }^{\circ}$ C 3253-2006, RUC No 06-2-0152725-0, seguidos ante el Tercer Juzgado de Familia de Santiago, caratulados "Vidal García-Huidobro Eugenia con Jano Bustamante Carlos, por sentencia de catorce de julio de dos mil ocho, se acogió, sin costas, la demanda de divorcio y, en consecuencia, se declaró terminado el matrimonio celebrado entre las partes el 22 de julio de 1983, por la causal de cese efectivo de la convivencia por más de tres años, ordenándose practicar la subinscripciones pertinentes. Se hizo lugar a la demanda reconvencional presentada por la cónyuge, sin costas, condenándose al demandado a pagar por ese concepto la suma de $\$ 70.000 .000$ (setenta millones), suma que el demandado deberá pagar de contado o mediante adjudicación a favor de la actora del bien raíz que actualmente le sirve de residencia.

Se alzó el demandado reconvencional y una de las salas de la Corte de Apelaciones de Santiago, por sentencia de cinco de noviembre de dos mil ocho, que se lee a fojas 272 de estos antecedentes, confirmó la de primer grado.

Respecto de esta última sentencia, el demandado reconvencional dedujo el recurso de casación en el fondo que pasa a analizarse.

Se ordenó traer los autos en relación.

\section{CONSIDERANDO:}

PRIMERO: Que el recurrente denuncia la infracción de los artículos 28 y 32 de la Ley 19.968, en relación con los artículos 61, 62, 63, 64, 65 y 66 de la Ley 19.947 e infracción a lo dispuesto en el artículo 19 del Código Civil, argumentando que los jueces del fondo han confundido el sistema de apreciación de la sana crítica con el de apreciación en conciencia, ya que no indican en cuál o cuáles de las máximas fundan su decisión, efectuando una mera enunciación genérica que en ningún caso constituye una ponderación real de la prueba. 
Indica que de haber analizado los sentenciadores debidamente la prueba rendida habrían arribado a la conclusión de que la actora no reúne los requisitos legales para ser merecedora de compensación económica o en su defecto y para el evento de considerarse que sí lo es, jamás se habría fijado un monto tal elevado.

Alega que escapa a toda lógica que el tribunal haya otorgado a título de compensación económica la señalada, puesto que ello, en definitiva, se sustenta en el presupuesto de que la actora debió percibir una remuneración mensual de $\$ 486.111$, lo que no resulta ajustado a la realidad y a las circunstancias del caso.

Por otro lado, señala que debe atenderse no sólo a la capacidad, preparación y profesión de la cónyuge, sino que también a lo que ésta podía y quería, en relación al ejercicio de una actividad lucrativa.

Expresa que no existe prueba en autos que permita decretar una compensación económica a favor de la actora, ni ningún elemento para regularla en la forma que se ha hecho. En efecto, sostiene que no se ha demostrado el menoscabo de la cónyuge, requisito fundamental para acceder a una pretensión de esta naturaleza, ni menos aún la relación de causalidad entre este elemento y el hecho de haberse dedicado al cuidado de la familia.

Indica que de haberse observado las normas citadas, sobre todo las que dicen relación con la ponderación de la prueba, conforme a la sana crítica, no se habría regulado la compensación económica en la cantidad fijada, debiendo haberse regulado en 144 ingresos mínimos mensuales, al valor que éstos tenían en las épocas en que éstos debieron haberse percibido, esto es, al equivalente al ingreso mínimo mensual remuneracional durante los años 1983 a 1995.

SEGUNDO: Que se han establecido como hechos en la causa los que siguen:

a) Las partes contrajeron matrimonio el 20 de julio de 1983, bajo el régimen de separación de bienes y tuvieron dos hijos, actualmente mayores de edad.

b) La vida en común de las partes se extendió desde el año 1983 hasta el año 2001, época en que terminó la convivencia.

c) Al momento de contraer matrimonio, la actora tenía sólo 21 años y se encontraba con cinco meses de embarazo.

d) Durante los años 1983 y 1995 la cónyuge no realizó actividad remunerada y se dedicó exclusivamente al cuidado de los hijos y a las labores propias del hogar común.

e) Al momento del matrimonio la cónyuge sólo había terminado su educación media y el cónyuge demandado no tenía el título profesional, recibiendo el de abogado en el año 1996.

f) Durante la vida en común y con posterioridad a la separación de las partes, la demandante ha prestado servicios esporádicos como arsenalera a favor de uno de sus hermanos. 
g) La actora registra exiguas cotizaciones y por cortos períodos de tiempo y no ha podido reinsertarse laboralmente en la forma que le permita vivir de acuerdo a su posición social.

TERCERO: Que sobre la base de los hechos anotados los sentenciadores concluyeron que la causal de divorcio esgrimida por el actor, esto es, el cese de convivencia por un plazo superior a tres años, se encuentra probada en autos, así, declararon terminado el matrimonio habido entre las partes. En cuanto a la demanda reconvencional, los sentenciadores señalaron los parámetros que para efectos de determinar la existencia y cuantía de la compensación económica menciona el legislador en el artículo 62 de la ley 19.947; ponderando la prueba aportada en conformidad a las normas de la sana crítica, asentaron los antecedentes fácticos precedentemente anotados y concluyeron que tanto la situación laboral y previsional de la cónyuge constituyen las áreas de mayor perjuicio, por lo que cuantificaron el menoscabo sufrido a partir de lo que ella dejó de ganar por no trabajar en el período reclamado, de acuerdo a un monto promedio de mercado, según actividades de la misma naturaleza.

CUARTO: Que el recurso de casación en el fondo es de derecho estricto y en él deben señalarse con precisión los errores de derecho que el recurrente advierte en el fallo impugnado, exigencia que no se cumple si los que se mencionan son contradictorios entre sí.

QUINTO: Que, en efecto, de la lectura del recurso en examen se advierte que se contienen en él planteamientos alternativos, esto es, llamados a regir sólo para el caso de que uno u otro no resulte acogido. El recurrente sostiene, en primer lugar, que la cónyuge no cumple el presupuesto esencial para obtener el derecho que reclama, cual es, haber sufrido un menoscabo económico como consecuencia de haberse dedicado, durante la vida matrimonial, al cuidado de los hijos y a las labores propias del hogar. Luego, plantea que yerran los sentenciadores al determinar el monto de la compensación económica, tanto en lo que dice relación con su base de cálculo, cuanto en la elevada suma fijada.

SEXTO: Que de acuerdo a lo anterior, es evidente que los argumentos del recurrente se contraponen entre sí, pues en la forma propuesta, por un lado desconoce el derecho de la actora reconvencional a la compensación económica demandada y, por otro, lo acepta, pues impugna no la existencia del derecho, sino su cuantía y forma de pago.

SÉPTIMO: Que el carácter confuso que el propio recurrente ha conferido a su libelo atenta contra la naturaleza del recurso intentado, de manera que no puede admitirse que se viertan en él reflexiones contradictorias, eventuales o para el supuesto de no prosperar determinado capítulo de impugnación ni menos puede aceptarse que se hagan peticiones opcionales que lo dejan, así, desprovisto de los requisitos que el artículo 772 del Código de Procedimiento Civil exige. Lo anterior se corrobora plenamente si se 
tiene presente, además, que el recurrente solicitó a esta Corte de Casación la dictación de una sentencia de reemplazo que rechace íntegramente la demanda reconvencional o, en su defecto, que reduzca ésta prudencialmente al equivalente a 144 ingresos mínimos mensuales, calculados al valor que éstos tenían en las épocas en que éstos debieron haberse percibido, esto es, al equivalente al ingreso mínimo mensual remuneracional durante los años 1983 a 1995, con costas.

OCTAVO: Que, sin perjuicio de lo anterior, cabe consignar que lo que el demandado ha pretendido con su recurso ha sido desvirtuar los hechos establecidos por los jueces del mérito, en circunstancias que conociendo la Corte Suprema de un recurso de casación en el fondo y de acuerdo a lo preceptuado en el artículo 785 del Código de Procedimiento Civil, debe aceptar como definitivos e inamovibles los hechos sentados por los jueces del mérito, salvo que se invoque y se acredite que en el establecimiento de estos se han vulnerado normas que gobiernan la prueba, lo que no ha ocurrido en la especie. En efecto, si bien el recurrente denunció como conculcada la norma contenida en el artículo 32 de la Ley 19.968, éste no explicó de qué forma ha sido infringida por los sentenciadores y cómo el supuesto error de derecho que reclama influyó en lo resolutiva de la sentencia atacada.

NOVENO: Que, por otra parte, cabe agregar que la actual Ley de Matrimonio Civil no define ni determina la naturaleza jurídica de la compensación económica, pero en su Capítulo VII, párrafo $1^{\circ}$, artículos 61 a 66, regula el régimen legal aplicable, señalando los presupuestos que la hacen procedente, los factores a tener en cuenta para su avaluación y la forma como debe fijarse.

DÉCIMO: Que, en el caso de autos, la dedicación al cuidado de los hijos y, como consecuencia de ello, el trabajo parcial desarrollado por la cónyuge se encuentran acreditados en el proceso de acuerdo a las conclusiones a las que han arribado los sentenciadores después de apreciar las probanzas.

UNDÉCIMO: Que otro requisito esencial para la procedencia de la compensación económica lo constituye la existencia de menoscabo económico en el cónyuge que la solicita y bien puede decirse que este elemento es el presupuesto de la acción. La ley no define el concepto, pero la interpretación armónica de los artículos de la Ley 19.947 que reglamentan la institución, permite concluir que se trata del efecto patrimonial que se produce en el cónyuge que no pudo trabajar o lo hizo en menor medida de lo que podía y quería. Este menoscabo debe ser probado por quien lo invoca en su favor y para ello el legislador se encargó de señalar algunas circunstancias que permitan determinar que ha existido tal menoscabo.

DUODÉCIMO: Que a falta de acuerdo entre las partes corresponde al juez de la causa establecer si se dan los requisitos que la institución exige y fijar su monto. De relacionar los artículos 63, 64, 65 y 66 de la ley 19.947, se infiere que el legislador ordena 
pagar un monto determinado invariable en el tiempo, cualesquiera sean las circunstancias personales y patrimoniales de los interesados -deudor o acreedor- posteriores a la sentencia que la regula. Si bien en esta materia el juez tiene discrecionalidad para fijar la cuantía de la compensación económica, se debe también considerar que el legislador estableció ciertos parámetros para ello, exponiendo criterios que dicen relación con el matrimonio, con el cónyuge deudor y con la situación personal del beneficiario.

DECIMOTERCERO: Que en los reproducidos fundamentos $21^{\circ}$ y $22^{\circ}$ del fallo de primer grado, los sentenciadores analizaron los factores propuestos en el artículo 62 de la Ley de Matrimonio Civil, dieron por establecido el menoscabo económico que significó para la actora su postergación laboral en beneficio del cuidado de los hijos. Para su avaluación, entregada al criterio del Tribunal, los jueces del grado explicaron la base de cálculo que estimaron prudente aplicar en la especie.

DECIMOCUARTO: Que, sobre el particular, es del caso hacer presente que la compensación económica no corresponde al valor exacto de lo que habría podido obtener el cónyuge beneficiario de haber trabajado o de haberlo hecho en mayor medida. En efecto, mediante esta institución no se trata de indemnizar la pérdida de una ganancia probable, es decir, su objetivo no es restituir lo perdido por su equivalente exacto y sólo se busca mitigar la situación económica desmedrada que afecta a quien tiene derecho a ella. Es el juez de la causa quien debe apreciar, en cada caso en particular, los criterios subjetivos del pasado de los cónyuges y las situaciones del futuro que el legislador sugiere para determinar su existencia y monto. En fin, verificado el cumplimiento de los presupuestos que la hacen procedente, esto es, acreditado en autos que se ha producido una situación injusta desde el punto de vista patrimonial para el cónyuge más débil de la relación matrimonial que termina, producida por la legítima opción de haberse dedicado al cuidado de los hijos y a las labores del hogar, corresponde a los jueces de la instancia fijar prudencialmente su monto.

DECIMOQUINTO: Que en estas condiciones, no puede sino concluirse que las normas sustantivas que se denuncian vulneradas no han sido infringidas por los sentenciadores, por el contrario, de acuerdo a los antecedentes fácticos asentados por los jueces del mérito, se aplicaron a una situación regida por ellas, pues probados los presupuestos que determinan el derecho demandado, es evidente que la fuerza jurídica de las reglas que regulan la institución no han sido desconocidas ni su interpretación contraria a la que procede.

DECIMOSEXTO: Que, por lo antes razonado, el recurso en estudio debe ser desestimado.

Por estas consideraciones y de acuerdo con lo previsto en los artículos 764, 765, 766, 767 y 768 del Código de Procedimiento Civil, se rechaza, sin costas, el recurso de casación 
en el fondo deducido por el demandado reconvencional a fojas 285 , contra la sentencia de cinco de noviembre de dos mil ocho, escrita a fojas 272 de estos antecedentes.

Redacción a cargo del Ministro señor Patricio Valdés Aldunate.

Regístrese y devuélvanse con sus agregados.

Pronunciada por la Cuarta Sala de la Corte Suprema integrada por los Ministros señores Patricio Valdés A., Pedro Pierry A., señora Gabriela Pérez P., y los Abogados Integrantes señores Roberto Jacob Ch. y Patricio Figueroa S. No firman el Ministro señor Pierry y el Abogado Integrante señor Figueroa, no obstante haber concurrido a la vista y al acuerdo de la causa, por estar ambos ausentes.

Autoriza la Secretaria de la Corte Suprema, señora Rosa María Pinto Egusquiza. $\mathrm{N}^{\circ}$ 904-09.

\section{COMENTARio}

Este comentario se centrará exclusivamente en el considerando noveno de la sentencia, no se efectuará un análisis de los otros considerandos ni de la parte resolutiva: demasiados ríos de tinta han corrido ya, sobre la compensación económica, como para incrementar más su torrentoso y turbio caudal.

Es usual en el ámbito del Derecho Civil, investigar la naturaleza jurídica de determinadas instituciones. En algunas ocasiones, estos intentos son infructuosos y los autores reconocen que ninguna de las alternativas es satisfactoria, llegando incluso a admitir que se trata de una "institución sui géneris". Sin embargo, pocas veces nos detenemos a examinar qué entendemos por la expresión "naturaleza jurídica" o, para plantearlo en términos más provocadores: “¿cuál es la naturaleza jurídica de la naturaleza jurídica?”. El planteamiento de la pregunta ya anticipa que con ello se busca simplemente algo inexistente.

Consideremos el caso más reciente en que se ha planteado este problema y al que se refiere la sentencia: la compensación económica se introdujo en el derecho nacional como una nueva institución. Sobre el problema de la determinación de la naturaleza jurídica de la compensación económica, don Hernán Corral señala: "Ante las dificultades que presenta la figura, quizá sea menester recurrir como criterio inicial a la función práctica para la cual fue diseñada. Pensar para qué se introdujo y el contexto social en el que se legisló. Antes de comenzar el examen de los textos normativos, convendría precisar, además, cuál fue el caso hipotético central que llevó a determinar la institución, dejando fuera del análisis otros supuestos a los cuales se extendió por razones diversas". ${ }^{1}$ Este trabajo del profesor Corral tiene el mérito de detenerse a analizar la función que cumple

${ }^{1}$ Corral Talciani, H., "La compensación económica en el divorcio y la nulidad matrimonial”, en Revista Chilena de Derecho, Pontificia Universidad Católica de Chile, vol. 34, No 1, 2007, pp. 23-40. 
esta herramienta teórica, al destacar que: "La cuestión no es irrelevante desde el punto de vista práctico. La identificación de la naturaleza jurídica sirve para dar contenido a las expresiones o conceptos de textura abierta contenidos en la regulación normativa, ayuda a elegir factores de decisión que aparecen aludidos únicamente por la intención del legislador de hacer enumeraciones de elementos no taxativas; y, finalmente, resulta útil para reconocer y aplicar el derecho supletorio". ${ }^{2}$

Más allá de la búsqueda de la naturaleza de las instituciones jurídicas se halla, en gran parte de las ocasiones, el afán de subsumir un determinado problema bajo un específico esquema normativo ideado inicialmente para casos diversos. Utilizar la técnica de la "naturaleza jurídica" contribuye a disfrazar la aplicación por analogía de otras normas del ordenamiento, lo cual conllevaría cambiar su extensión, argumentando que habría una "esencia" común entre otros casos expresamente regulados por el legislador y las nuevas situaciones que van surgiendo, debido a lo cual, éstas habrían sido considerados implícitamente en la regulación legal. ${ }^{3}$ Los tenaces trabajos de los juristas por desentrañar la naturaleza jurídica de una determinada institución se encuentran indefectible y terminantemente condenados a fracasar desde sus inicios. Entre otras consideraciones, debido a que lo buscado, tal como se lo persigue, no tiene existencia. Un destacado lógico del derecho como Bulygin ha enfatizado que términos como "matrimonio" o "posesión" poseen un sentido únicamente en el marco de cierto contexto. Inquirir por una supuesta naturaleza o esencia de la estipulación a favor de otro o de la compensación económica, simplemente es un absurdo. ${ }^{4}$

La utilización de la técnica de la "naturaleza jurídica" encubre que nos enfrentamos a una decisión acerca de la forma en que debemos comprender convencionalmente cierto texto. Esta decisión es adoptada por el mismo intérprete, de acuerdo a sus preferencias y tomando en cuenta múltiples consideraciones tales como la conciencia jurídica material, los factores pragmáticos de interpretación, la búsqueda de un significado razonable, etc. Por eso no sorprende que los autores difieran enormemente respecto a cuál sea la naturaleza jurídica de una institución en particular.

Manuel Atienza, en sus "Diez Consejos para escribir un buen trabajo de dogmática”, expresa: “...muchos pseudoproblemas -especialmente, si han sido con asiduidad objeto de reflexión dogmática- pueden esconder un problema genuino que se pone al descubierto cuando se lo enfoca desde el ángulo adecuado. Por ejemplo, cuando lo que esté en cuestión sea la "naturaleza jurídica" de la institución X, no enfoque el problema como si se tratara de descubrir la "verdadera esencia" de X. Por el contrario, comience por indagar qué consecuencias tendría el que a $\mathrm{X}$ se le califique de $\mathrm{Y}$ o de Z. Luego trate

\section{Ibídem.}

${ }^{3}$ Nino, C.S., Introducción al Análisis del Derecho, Barcelona, Editorial Ariel, $11^{\text {a }}$ edición, 2003, pp. 315 y ss.

${ }^{4}$ Haba, E. P., Metodología Jurídica Irreverente, Editorial Dykinson, Madrid, 2006, pp. 207-211. 
de justificar por qué es preferible un tipo de consecuencias a otro. Cuando haya hecho esto último, habrá resuelto ya el problema de la naturaleza jurídica de X".5

¿Qué se busca al indagar sobre la naturaleza jurídica? Las posibilidades son varias, ya que esta expresión es usada con propósitos diversos:

a) determinar el régimen jurídico aplicable a una institución, a fin de establecer cuáles son sus consecuencias jurídicas. Ello implica efectuar una operación de calificación jurídica.

b) identificar los elementos que la constituyen, es decir, cuáles son sus hechos condicionantes.

c) precisar los requisitos o condiciones que deben reunirse, según el uso, para emplear la palabra.

d) en otros casos, por ejemplo en la compensación económica, precisar los criterios de determinación del quántum.

e) en algunas ocasiones, determinar la validez y jerarquía de una norma jurídica (de acuerdo a las normas que rigen la producción jurídica).

f) dar contenido a un concepto jurídico indeterminado.

g) de modo más general que el anterior, emplear los espacios de discrecionalidad que concede el Derecho.

En la mayoría de estas situaciones se procura integrar un vacío o laguna en el ordenamiento. ¿Por qué es deficiente como técnica de argumentación? Porque puede implicar un ardid retórico: encubre esta actividad de integración, presentándola como una mera descripción. La doctrina, en algún sentido, está "creando" derecho (es lo que corresponde en estos casos), el problema está en que reclama para ello una validez absoluta. Y carecemos de un procedimiento intersubjetivo para verificar la corrección de esa conclusión (para captar esa naturaleza jurídica).

Para aclarar debidamente las implicancias de la determinación de la naturaleza jurídica de una institución, resulta sumamente didáctico recordar el problema al que se enfrentaron los naturalistas del siglo XVIII, para clasificar a una nueva especie descubierta: el ornitorrinco. "No hacía tantos años que Linneo había establecido en su Sistema Natural (1755) la clasificación vigente de los animales, y el lugar que en ella ocupaba la clase de los mamíferos no había hueco alguno para semejantes extravagancias. Los naturalistas acabaron por crear un nuevo orden para él, el de los monotremas, pero estuvieron discutiendo todos esos años si tal orden nuevo pertenecía a la clase de las aves o a la de los mamíferos. Esta discusión desbordó hasta tal punto los límites de la ciencia natural que acabó por llegar hasta el mismísimo pensamiento jurídico, y tuvo un sorprendente eco en los escritos de uno de los más importantes juristas del siglo XIX”. 6

${ }^{5}$ Atienza, M., "Diez Consejos para escribir un buen trabajo de dogmática” en Isonomía: Revista de Teoría y Filosofía del Derecho, Instituto Tecnológico Autónomo de México, núm. 3, octubre de 1995, p. 224.

${ }^{6}$ Laporta, F. J., "El ornitorrinco y el Consejo de Estado", publicado en la sección "Opinión”, del diario español El País, el 1 de febrero de 2005. 
Así vistas las cosas, varios siglos después, la doctrina civil chilena ya ha encontrado su propio ornitorrinco: la compensación económica. Llama la atención la gran cantidad de trabajos y fallos de los tribunales que se pronuncian acerca de la naturaleza jurídica de esta nueva institución, asumiendo las más variopintas posiciones. Esto, al menos, nos debe hacer pensar acerca de la manera en que se utiliza este procedimiento teórico, y acerca de su verdadera utilidad y rigor, ya que puede servir prácticamente para dar fundamento a cualquier solución.

Así, esta sentencia de la Corte Suprema, en su considerando noveno, hace bien al no embarcarse en la vana búsqueda de la naturaleza jurídica de la compensación económica. Para analizar el resto del problema, basta con recurrir a la copiosa y ampliamente conocida bibliografía publicada sobre el punto. 